CERN-TH/95-82

MC-TH-96/05

UCL/HEP 96-02

\title{
Multiparton Interactions in Photoproduction at HERA
}

\author{
J. M. Butterworth ${ }^{1}$, J. R. Forshaw ${ }^{2}$ and M. H. Seymour ${ }^{3}$ \\ ${ }^{1}$ University College London, Gower St., London WC1E 6BT, UK. \\ ${ }^{2}$ University of Manchester, Oxford Rd., Manchester, M13 9PL, UK.

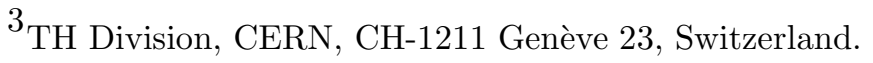

$29^{\text {th }}$ Jan. 1996

\begin{abstract}
The high energy photoproduction of jets is being observed at the ep collider, HERA. It may be that the HERA centre-of-mass energy is sufficiently large that the production of more than one pair of jets per ep collision becomes possible, owing to the large number density of the probed gluons. We construct a Monte Carlo model of such multiparton interactions and study their effects on a wide range of physical observables. The conclusion is that multiple interactions could have very significant effects upon the photoproduction final state and that this would for example make extractions of the gluon density in the photon rather difficult. Total rates for the production of many (i.e. $\geq 3$ ) jets could provide direct evidence for the presence of multiple interactions, although parton showering and hadronization significantly affect low transverse energy jets.
\end{abstract}




\section{Introduction}

In recent years, the TEVATRON $(\bar{p} p)$ and HERA $(e p)$ colliders have made it possible to study the standard theory of strong interactions (QCD) in a new regime: the regime of high parton densities. QCD predicts a rapid build up of very slow gluons within hadrons, which can be observed at high centre of mass $(\mathrm{CM})$ energies, i.e. $x \sim Q^{2} / s \ll 1$ where $s$ is the CM energy and $Q^{2}$ is the appropriate hard scale. Both the TEVATRON and HERA can study this region in some detail, through the production of high $p_{T}$ jets, heavy flavours and large- $t$ diffractive scattering. At HERA, we have the additional possibility of studying this 'small- $x$ ' physics using deep inelastic scattering (where $x=x_{\mathrm{Bj}}$ ).

In this paper, we study jet production at HERA in those events where the photon is nearly real (photoproduction). Photoproduction of jets has been observed at HERA [1] and different components of the cross section identified [2]. The components are easy to define in leading order QCD and are termed direct and resolved. In the direct sector, the photon carries all of its energy into the hard scatter whilst in the resolved sector, only a fraction of the photon energy participates in the hard subprocess. At higher orders this simple distinction between direct and resolved is no longer uniquely defined. However, a precise definition of the separation between resolved and direct processes in terms of physical observables is possible [3] allowing the effects of the parton distributions in the proton and photon to be disentangled [ 4 . This has already been implemented in higher order dijet cross section calculations [5].

The total rate is high for the photoproduction of jets and allows a study of small $x$ phenomena when $x \sim E_{T}^{\text {jet }}{ }^{2} / s \ll 1\left(E_{T}^{\text {jet }}\right.$ is the jet transverse momentum $)$. Naively, one might conclude that the $1800 \mathrm{GeV}$ CM energy available at the TEVATRON would make it far superior to HERA (with a $\gamma p \mathrm{CM}$ energy that is typically $\simeq 200 \mathrm{GeV}$ ) regarding the study of small $x$ physics through jet production. However, this is not the case. Associated with higher CM energies is an increase in the background of soft physics. At HERA, the asymmetric configuration of the lab frame (27 GeV leptons collide with $820 \mathrm{GeV}$ protons) tends to boost the poorly understood 'soft' physics into the proton direction and hence down the 'forward' beam hole. The asymmetric boost of the $\gamma p$ system in the lab also means that jets that have been produced by small $x$ partons within the proton appear in the central region in the lab frame of reference and are thus clearly visible in the detectors. None of these benefits are present at the TEVATRON, where the lab frame is also the $p \bar{p} \mathrm{CM}$ frame.

Due to the proliferation of low $x$ partons (which can be inferred from the strong growth observed in the HERA data on the proton structure function [6]), it is possible that more than one pair of jets can be produced per $\gamma p$ collision. This multiple (parton) scattering, illustrated in figure 1, is expected at high enough CM energies and signals the onset of unitarization corrections to the simple perturbative QCD picture of $2 \rightarrow 2$ parton scattering. Unitarization corrections are certainly necessary since the total cross section for inclusive jet photoproduction (calculated in lowest order QCD with steeply rising parton distribution functions) will ultimately exceed the total $\gamma p$ cross section'. As we shall see, this apparent anomaly is resolved once we appreciate that the inclusive jet cross section exceeds the total cross section for jet production by a factor equal to the mean multiplicity of multiple interactions. We introduce an eikonal model which

\footnotetext{
${ }^{1}$ Multiple scattering is only one manifestation of the unitarization corrections that are expected to occur at high energies. The steepness of the parton densities will eventually be tamed by additional corrections. We ignore such effects in this paper.
} 


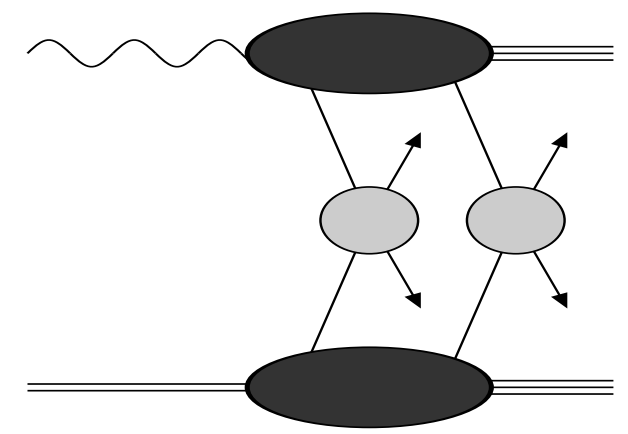

Figure 1: An example of a multiple scattering in a $\gamma p$ collision.

assumes that individual hard scatters are uncorrelated. This allows us to model the rate of multiple interactions and study their effects upon the hadronic final state. For the latter study, it is most convenient to subject our model to a Monte Carlo simulation and this will allow us to make full use of the available and forthcoming HERA data.

In section 2, we describe the eikonal model of refs. [7, 8, 9] and show how it predicts a significant rate for the production of multijet events at HERA energies. Also described here is the implementation of the formalism within the HERWIG Monte Carlo package [10]. By integrating our formalism within HERWIG, we can make realistic studies of the final state which include the effects of parton showering and hadronization. In section 3 a number of key photoproduction distributions are presented for our default model, and compared to the results obtained without multiple interactions. In sections 4 and 5 , we examine the effects of using different parton distribution functions and of variations on the default model. In section 6, comparisons are made to available HERA data. We show that multiple scattering can be expected to make a clean extraction of parton distribution functions in the photon rather difficult.

\section{Why Multiple Interactions at HERA?}

We are interested in jet production in $\gamma p$ reactions and at the level of the hard subprocess we assume that this can be approximated by the lowest order matrix element for $2 \rightarrow 2$ parton scattering with final state partons produced with a transverse momentum, $p_{T}>p_{T}^{\min } \gg \Lambda_{\mathrm{QCD}}$. Jets can be produced directly via the $\gamma$-parton hard subprocess or indirectly via partons from the resolved photon scattering with partons in the proton. These resolved partons can be generated either non-perturbatively (i.e. the $\gamma$ splits into a large size $q \bar{q}$ pair) or perturbatively (i.e. via perturbative evolution of a small size $q \bar{q}$ pair). Let us start by considering a $\gamma p$ interaction at some fixed centre-of-mass energy, $s_{\gamma p}$. In the CM frame we think of the proton and resolved photon as Lorentz contracted 'parton pancakes' colliding at some impact parameter, $b$. The mean number of jet pairs produced in this resolved- $\gamma-p$ interaction is then

$$
\langle n(b, s)\rangle=\mathcal{L}_{\text {partons }} \otimes \hat{\sigma}_{H}
$$

where $\mathcal{L}_{\text {partons }}$ is the parton luminosity and $\hat{\sigma}_{H}$ is the cross section for a pair of partons to produce a pair of jets (i.e. partons with $p_{T}>p_{T}^{\min }$ ). The direct photon interaction generates 
only a single pair of partons and we ignore it for now (it will be included as a separate hard subprocess in the Monte Carlo simulation).

The convolution is because the parton cross section depends upon the parton energies. More specifically,

$$
d \mathcal{L}_{\text {partons }}=A(b) n_{\gamma}\left(x_{\gamma}\right) n_{p}\left(x_{p}\right) d x_{\gamma} d x_{p}
$$

where $n_{i}\left(x_{i}\right)$ is the number density of partons in hadron $i$ which carry a fraction $x_{i}$ of the hadron energy. For ease of notation we do not distinguish between parton types and have ignored any scale dependence of the number densities. $A(b)$ is a function which specifies the distribution of partons in impact parameter. It must satisfy

$$
\int \pi d b^{2} A(b)=1
$$

in order that the parton luminosity integrated over all space is simply the product of the parton number densities. Factorizing the $b$ dependence like this is an assumption. In particular we do not contemplate QCD effects which would spoil this, e.g. perhaps leading to 'hot spots' of partons. For the proton, the number density is none other than the proton parton density, i.e. $n_{p}\left(x_{p}\right) \equiv f_{p}\left(x_{p}\right)$. The number density $n_{\gamma}$ of partons given that the photon is resolved is related to the photon parton density by a factor of $\sim \alpha_{\mathrm{em}}$, i.e. $n_{\gamma}\left(x_{\gamma}\right) \sim f_{\gamma}\left(x_{\gamma}\right) / \alpha_{\mathrm{em}}$. In our model, we ignore multiple scattering which arises due to interactions of the small size fluctuations of the incoming photon and therefore assume that the photon interacts just like a hadron. The large size hadronic fluctuations of the photon can be modelled assuming vector meson dominance and allow us to estimate that $n_{\gamma}\left(x_{\gamma}\right)=f_{\gamma}\left(x_{\gamma}\right) / P_{\text {res }}$ where $P_{\text {res }}=\kappa 4 \pi \alpha_{\text {em }} / f_{\rho}^{2}$. The sum over hadronic fluctuations determines $\kappa$. Unless otherwise stated, we assume $\rho$-dominance, i.e. $\kappa=1$ and $P_{\text {res }} \approx 1 / 300$.

Thus, after performing the convolution, we can write:

$$
\left\langle n\left(b, s_{\gamma p}\right)\right\rangle=\frac{A(b)}{P_{\text {res }}} \sigma_{H}^{\text {inc }}\left(s_{\gamma p}\right),
$$

where $\sigma_{H}^{\text {inc }}\left(s_{\gamma p}\right)$ is the inclusive cross section for $\gamma p \rightarrow$ jets. Restoring the parton indices, it is given by

$$
\sigma_{H}^{\mathrm{inc}}\left(s_{\gamma p}\right)=\int_{p_{T}^{\min 2}}^{s_{\gamma p} / 4} d p_{T}^{2} \int_{4 p_{T}^{2} / s_{\gamma p}}^{1} d x_{\gamma} \int_{4 p_{T}^{2} / x_{\gamma} s_{\gamma p}}^{1} d x_{p} \sum_{i j} f_{i / \gamma}\left(x_{\gamma}, p_{T}^{2}\right) f_{j / p}\left(x_{p}, p_{T}^{2}\right) \frac{d \hat{\sigma}_{i j}\left(x_{\gamma} x_{p} s_{\gamma p}, p_{T}\right)}{d p_{T}^{2}} .
$$

In order to investigate further the structure of events containing multiple interactions we need to know the probability distribution for having $m$ (and only $m$ ) scatters in a given resolved $-\gamma-p$ event, $P_{m}$. In order to do this we assume that the separate scatters are uncorrelated, i.e. they obey Poissonian statistics. Thus

$$
P_{m}=\frac{\left(\left\langle n\left(b, s_{\gamma p}\right)\right\rangle\right)^{m}}{m !} \exp \left(-\left\langle n\left(b, s_{\gamma p}\right)\right\rangle\right) .
$$

This formula is central to the Monte Carlo implementation in HERWIG.

We can now ask for the total cross section for $\gamma p \rightarrow$ partons with $p_{T}>p_{T}^{\min }$.

$$
\begin{aligned}
\sigma_{H}\left(s_{\gamma p}\right) & =\pi P_{\text {res }} \int d b^{2} \sum_{m=1}^{\infty} P_{m} \\
& =\pi P_{\text {res }} \int d b^{2}\left[1-\exp \left(-\left\langle n\left(b, s_{\gamma p}\right)\right\rangle\right)\right]
\end{aligned}
$$


Since the total inclusive cross section $\left(\sigma_{H}^{\text {inc }}\right)$ counts all jet pairs (even ones which occur in the same event) we expect it to be larger than $\sigma_{H}$ by a factor equal to the mean number of multiple interactions per event (i.e. averaged over impact parameter). This is easy to see. Let $\left\langle n\left(s_{\gamma p}\right)\right\rangle$ be the average number of jet pairs produced in resolved $-\gamma-p$ events which contain at least one pair of jets, then

$$
\begin{aligned}
\left\langle n\left(s_{\gamma p}\right)\right\rangle & =\frac{\int d b^{2} \sum_{m=1}^{\infty} m P_{m}}{\int d b^{2} \sum_{m=1}^{\infty} P_{m}} \\
& =\frac{\int d b^{2}\left\langle n\left(b, s_{\gamma p}\right)\right\rangle}{\int d b^{2}\left[1-\exp \left(-\left\langle n\left(b, s_{\gamma p}\right)\right\rangle\right)\right]} \\
& =\frac{\sigma_{H}^{\mathrm{inc}}\left(s_{\gamma p}\right)}{\sigma_{H}\left(s_{\gamma p}\right)} .
\end{aligned}
$$

Note that $\sigma_{H}$ must always be less than the total $\gamma p$ cross section, whereas $\sigma_{H}^{\text {inc }}$ need not be.

Jet cross sections at HERA are sensitive to the proton parton density down to small momentum fractions, $x \sim 10^{-3}$. As shown by the measurement of the structure function in deep inelastic scattering at HERA, this is the region of high parton density (which rises as $x^{-0.3}$ at $Q^{2} \sim$ $10 \mathrm{GeV}^{2}$ [6]). For $p_{T}^{\min } \sim 1-2 \mathrm{GeV}$, one expects that $\sigma_{H}^{\text {inc }}(1 \mathrm{TeV}) \sim 1 \mathrm{mb}$, which is much larger than the total $\gamma p$ cross section, which is $\sim 200 \mu \mathrm{b}$ according to the successful [11] Regge pole model of Donnachie and Landshoff [12]. This suggests the possibility that $\left\langle n\left(s_{\gamma p}\right)\right\rangle$ is significantly greater than 1.

To complete the description of our model, we must now specify how the partons are distributed in impact parameter space. As a first approximation, we ignore pQCD effects and assume that $A(b)$ can be written as a convolution of form factor distributions, i.e. starting from

$$
\begin{aligned}
G_{p}(\mathbf{b}) & =\int \frac{d^{2} \mathbf{k}}{(2 \pi)^{2}} \frac{\exp (\mathbf{k} \cdot \mathbf{b})}{\left(1+\mathbf{k}^{2} / \mu^{2}\right)^{2}} \\
G_{\gamma}(\mathbf{b}) & =\int \frac{d^{2} \mathbf{k}}{(2 \pi)^{2}} \frac{\exp (\mathbf{k} \cdot \mathbf{b})}{1+\mathbf{k}^{2} / \nu^{2}}
\end{aligned}
$$

with $\mu^{2}=0.71 \mathrm{GeV}^{2}$ and $\nu^{2}=0.47 \mathrm{GeV}^{2}$, we can write

$$
A(b)=\int d^{2} \mathbf{b}^{\prime} G_{p}\left(\mathbf{b}^{\prime}\right) G_{\gamma}\left(\mathbf{b}-\mathbf{b}^{\prime}\right) .
$$

The integral then yields [9]:

$$
A(b)=\frac{1}{2 \pi} \frac{\mu^{2} \nu^{2}}{\mu^{2}-\nu^{2}}\left[\frac{\mu^{2}}{\mu^{2}-\nu^{2}}\left(K_{0}(\nu b)-K_{0}(\mu b)\right)-\frac{\mu b}{2} K_{1}(\mu b)\right] .
$$

The formalism just described has been discussed extensively in the literature (see ref. [13, 14]) but largely in the context of total cross sections. The expected rates for multi-jet processes have also been studied [15]. Owing to the complexity of the final state, in which many jet pairs may be produced, previous studies have been confined to rather inclusive properties of the model. To really test for the existence of multiple interactions however, it is necessary to confront the vast amount of data that HERA is making available with more detailed properties of the final state and to this end we have constructed a Monte Carlo simulation program. In 
ref. [16] some results of an earlier version of this simulation were presented. In this paper we present a more comprehensive study of the effects of this model on the photoproduction final state. The program is written as an extension to the HERWIG [10] package and can also be used to simulate the effects of multiple interaction in other processes, such as $\gamma \gamma$ interactions in $e^{+} e^{-}$collisions [17]. There is a degree of overlap with the multiple interaction formalism that is included in the PYTHIA program 18.

The effect of eikonalization on the total $\gamma-p$ cross section is shown in figure 2a. In figure 2 $2 \mathrm{~b}$ this is translated into the effect on the differential ep cross section $d \sigma_{e p} / d y$ where $s_{\gamma p}=4 y E_{e} E_{p}$ ( $E_{e}$ is the incoming electron energy and $E_{p}$ the incoming proton energy). The $p_{T}^{\min }$ is taken to be $3 \mathrm{GeV}$ and $P_{\text {res }}=1 / 300$. The parton distribution sets used are the GS2 set [19] for the photon and the GRV [20] set for the proton. Clearly (for this choice of parameters) the effect of multiple scattering is to significantly lower the cross section, with significance increasing with increasing $s_{\gamma p}$ (and therefore $y$ ). This is reflected by a significant probability for more than one hard interaction in a $\gamma p$ event, as shown in figure 2 $2 \mathrm{c}$, where it can be seen that, depending upon $y$, as many as 10-15\% of $\gamma p$ events contain more than one parton-parton scattering. This is expressed as a differential cross section for $N$ and only $N$ scatters in figure 2 $2 \mathrm{~d}$. Note that the hard cross section for $\sqrt{ } s_{\gamma p} \approx 200 \mathrm{GeV}$ represents around $20 \%$ of the total $\gamma$ - $p$ cross section [11].

\subsection{Monte Carlo Implementation}

The cross sections illustrated in figure 2 indicate that the effects of multiple interactions might be observable at HERA. However, the experiments do not measure jet cross sections as low as $E_{T}^{\text {jet }} \approx 3 \mathrm{GeV}$. The lowest $E_{T}^{\text {jet }}$ cut used on measured cross sections so far has been $6 \mathrm{GeV}$. The smearing of jet energies due to parton showering and hadronization effects mean that a $p_{T}^{\min }$ cut of around $2.5 \mathrm{GeV}$ is often used by the experiments in Monte Carlo simulations to obtain a good description of the data at higher $E_{T}^{\text {jet }}$ cuts. The pile-up of more than one hard scatter in an event can be expected to increase these migrations, and in order to study the effect of multiple interactions on real jets rather than partons, it is necessary to include realistic hadronization and parton showering models in conjunction with the eikonal model described above. To this end, the HERWIG Monte Carlo program was adapted to allow the generation of multiple hard subprocesses in a single hadron-hadron, photon-hadron or photon-photon event.

In the default version of HERWIG, event generation begins with the hard process, QCD dijet production in our case. This is generated according to the lowest order cross section using whichever parton distributions have been selected by the user. Associated with the hard process is a hard scale, $Q$ ( $\sim E_{T}^{\text {jet }}$ for dijet production), and the cross section for additional partons to be radiated in the same event is enhanced by powers of $\log Q / Q_{0}$ at each order in $\alpha_{s}$, where $Q_{0}$ is a lower cutoff defining the resolvability of partons. In order to fully describe the final state, $Q_{0}$ is required to be around the typical hadronic scale, $Q_{0} \sim 1 \mathrm{GeV}$, and the logarithmic terms are large. Therefore such terms must be summed to all orders in $\alpha_{s}$. This summation can be implemented as a probabilistic evolution of the external (both incoming and outgoing) partons. Any parton shower algorithm that implements the DGLAP splitting functions [21] correctly resums the single logarithmic terms associated with the collinear emission that drives fragmentation function and parton distribution function evolution, but to fully model the final state, single and double logarithmic terms associated with soft emission must also be summed 

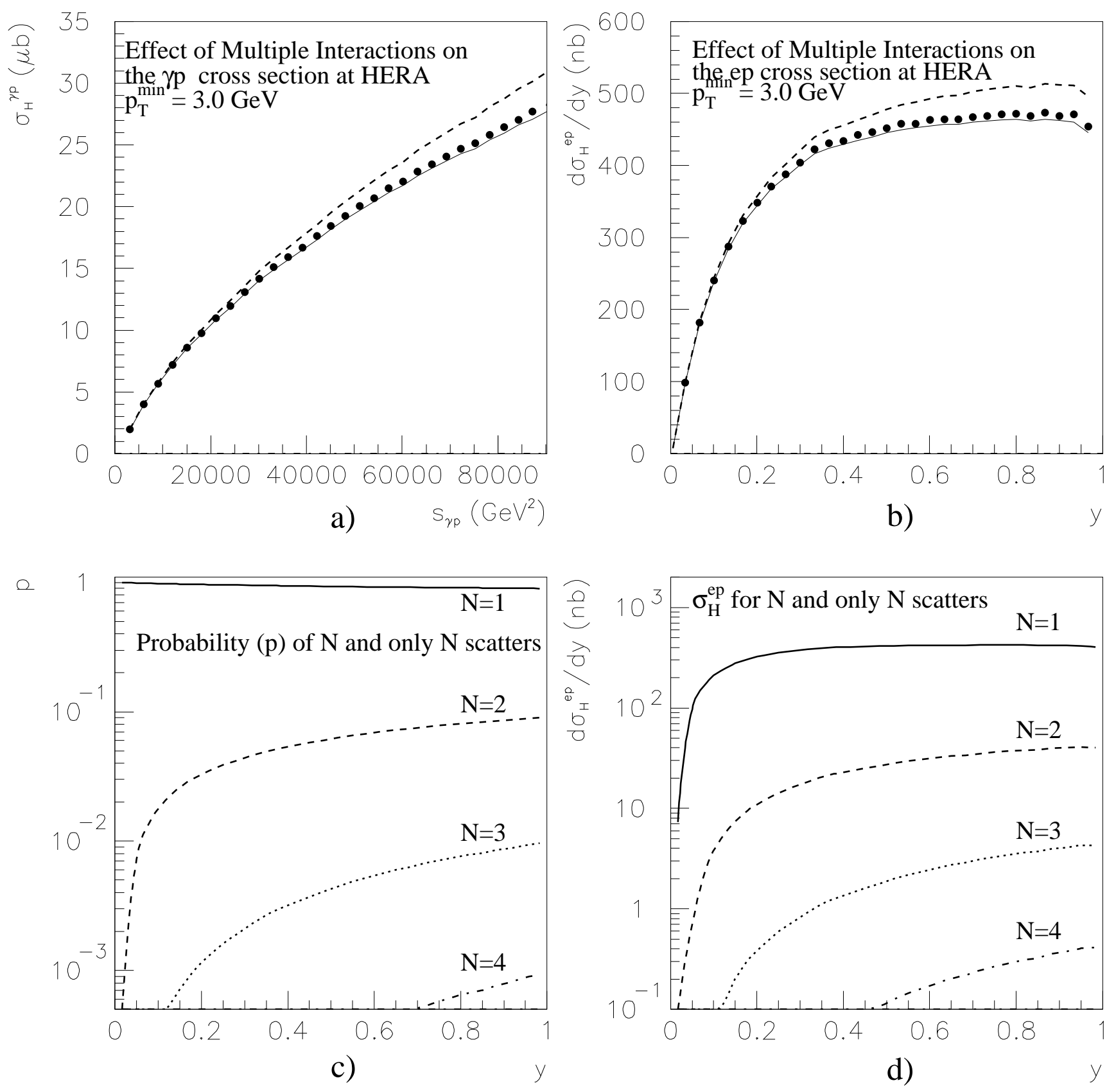

Figure 2: a) $\sigma_{H}$ as a function of $s_{\gamma p}$, the squared c.m. energy of the photon-proton system. b) $d \sigma_{H}^{e p} / d y$ as a function of $y$. In a) and b) the dashed lines show the result with no multiple interactions, the solid lines show the result of including multiple interactions and the points indicate the cross sections actually generated by the Monte Carlo program (see text). In c) the probability of $N$ and only $N$ scatters as a function of $y$ is shown. In d) the cross section for $N$ and only $N$ scatters as a function of $y$ is shown. 
to all orders. This is done by coherent parton shower algorithms [22], which also implement the DGLAP splitting functions, but with specific choices for the definitions of evolution scale and parton resolution criterion. For final-state partons, the algorithm evolves a single hard parton to many softer ones. For initial-state partons, the evolution is 'backwards', from the hard process to the incoming hadron or photon, evolving a single incoming parton to another at larger $x$, together with many additional outgoing partons. The backward evolution algorithm [23] ensures that at each stage of the evolution, the distribution of parton $x$ values agrees with that of the input distribution function. For the photon, the inhomogeneous term in the evolution equation appears as an additional vertex in the backward evolution, giving the possibility that it will terminate 'anomalously', with the incoming photon giving all of its energy to the hard process and its associated parton shower.

Finally, after perturbative parton evolution, a non-perturbative model of the transition from partons to hadrons is invoked. In HERWIG, a simple model that makes use of the preconfinement property of QCD is used. At leading order in the number of colours, the structure of a parton shower is such that each parton ends up close, in both momentum-space and real-space, to a parton that carries the opposite colour quantum numbers. The model therefore combines each such colour-connected pair of partons to form a single colourless cluster. This is assumed to decay directly to hadrons, with the choice of hadron species controlled purely by phase-space arguments [24]. This model therefore has few adjustable parameters, which are already rather well constrained by data from $\mathrm{e}^{+} \mathrm{e}^{-}$annihilation. For processes with incoming partons, there is an additional freedom, of how to model the break-up of the incoming hadron or photon after one parton is taken from it. In HERWIG, this is done by requiring that the backward evolution results in a valence parton $(\mathrm{u}, \mathrm{d}$ and $\mathrm{s}$ quarks and antiquarks are all considered valence partons of a photon). If the perturbative evolution did not end anomalously, then additional emission is generated below $Q_{0}$, but still according to the perturbative distributions, to obtain a valence parton. Once the 4-momentum of this valence parton is known, energy-momentum conservation is used to calculate the momentum of the remaining hadron or photon remnant. For photons, the flavour of the remnant is simply that of the antiparticle of the valence parton, while for protons, the remaining diquark is taken to be a single anticoloured parton. The cluster containing the remnant parton is then hadronized like any other. Clearly this step is unnecessary in events where the backward evolution terminated anomalously, since there is no remnant parton in that case.

When incorporating our multiple interactions model into the Monte Carlo event generator several additional problems arise from the fact that the model assumes that different scatterings are uncorrelated, while in the event generator this is not possible. The first is that of energy conservation. In the analytical model, there is a non-zero (though small) cross section for events in which the total energy participating in hard scatters is greater than the energy of the incoming particles. This is exacerbated by the backward evolution, since additional energy is radiated away in the additional partons. We impose global energy-momentum conservation by adding the simplest possible correlation between scatters: a $\Theta$-function in the cross section to produce $m$ and only $m$ scatters requiring their total energy to be less than the available energy. This will reduce the amount of eikonalization of the cross section relative to the analytical model. When generating events, this is implemented by calculating the total cross section and probabilities for exactly $m$ scatters using the analytical model, and generating hard scatters according to these probabilities. Scatters in which energy-momentum cannot be conserved are then vetoed (although the event itself is kept, with all the other scatters). At the end of event 
generation, the number of vetoed scatters is used to make a revised estimate of the total cross section.

In the analytical model, only events in which the photon is resolved are eikonalized, while direct photon events are not. However, events in which the backward evolution terminated anomalously should also be called direct in this context (they correspond to the small size fluctuations of the photon) and should not be eikonalized. Therefore, if the backward evolution does terminate anomalously, no further scatters are allowed in that event. This is treated just like the vetoing from energy conservation so also revises the final estimate of the total cross section. Since the separation into the anomalous and resolved parts of the distribution function is model dependent, we provide an option that enables multiple interactions in anomalous events, so that one can gauge the relative importance of this feature of the model.

These revised cross sections are shown as data points on figure $2 \mathrm{a}$ and b.

The simple model used in HERWIG for the hadron and photon remnants is no longer appropriate when there is more than one scatter. This is because once the incoming colourless hadron has been replaced by an outgoing coloured remnant by the first interaction, the same procedure cannot be iterated for subsequent interactions. Instead, we label the remnant as a new kind of incoming hadron for the other interactions, which has identical properties to the original hadron, except that gluons are labelled as its valence partons. Thus, if the backward evolution does not result in a gluon, an additional emission is forced to produce one. The outgoing coloured remnant from this interaction is the same as that from the first, but with reduced momentum. This procedure can then be iterated as many times as required for all subsequent interactions. By thus modifying only the part of the model that connects the hard processes to the incoming hadron, and not the backward evolution itself, we ensure the predictivity of the model, using the parameters fixed to other reactions.

\section{Effect on the Hadronic Final State}

The jet properties of the final state are of great importance in understanding the underlying QCD processes, and the possible influence of multiple scattering needs to be carefully examined. For example, jet rates in photoproduction may well lead to important information regarding the gluon content of the photon and the proton [1, 14. In this section we examine the general effects of multiple scattering on the properties of the final state measurable at HERA. In order to do this, we take a default set of parameters chosen where appropriate to reflect published data from the HERA experiments and the best available theoretical estimates. These choices are as follows:

- $P_{\text {res }}=1 / 300$ : This is motivated by assuming $\rho$-dominance.

- $p_{T}^{\min }=3.0 \mathrm{GeV}$ : This is consistent with the values typically used by the experiments to describe their data at $E_{T}^{\text {jet }} \geq 6 \mathrm{GeV}$.

- The GRV parton distribution set is used for the proton [20]. This set is the result of a global analysis including the HERA measurements of the proton structure function $F_{2}$ [6]. 
- The GS2 parton distribution set is used for the photon [19]. The gluon distribution in the photon is poorly constrained over the region studied (the desire to constrain the gluon is a major motivation for measuring high- $E_{T}$ photoproduction cross sections at HERA) and there is little reason to favour any one out of several available parameterizations.

- A cut on the $\gamma p \mathrm{CM}$ energy was made, $114 \mathrm{GeV} \leq \sqrt{ } s_{\gamma p} \leq 295 \mathrm{GeV}$, similar to those usually made by the experiments.

These choices define what we call our 'default' model. In the subsequent sections the sensitivity to various choices made here will be examined and discussed. Events were generated both with and without multiple interactions. All other parameters of the Monte Carlo model were left at their default values, which are tuned to $\mathrm{e}^{+} \mathrm{e}^{-}$data and give a good description of the direct component of photoproduction at HERA [3].

The initial expectation is that each pair of jets will be produced back-to-back azimuthally with balancing transverse momenta. For two-to-two parton scattering in leading order QCD energy and momentum conservation gives

$$
x_{p}^{\mathrm{LO}}=\frac{\sum_{\text {partons }}\left(E+p_{z}\right)_{\text {parton }}}{2 E_{p}}, x_{\gamma}^{\mathrm{LO}}=\frac{\sum_{\text {partons }}\left(E-p_{z}\right)_{\text {parton }}}{2 y E_{e}} .
$$

The proton direction defines the positive $z$-axis, $E_{e}$ and $E_{p}$ are the initial lepton and proton energies and the sum is over the two final state partons. For direct photon events, $x_{\gamma}^{\mathrm{LO}}=1$. Since it is not possible to measure partons an observable has been defined by ZEUS [3] in terms of jets which is analogous to $x_{\gamma}^{\mathrm{LO}}$. This observable, called $x_{\gamma}^{\text {obs }}$, is the fraction of the photon's momentum which emerges in the two highest $E_{T}^{\text {jet }}$ jets. The explicit definition is,

$$
x_{\gamma}^{\mathrm{obs}}=\frac{\sum_{\mathrm{jets}} E_{T}^{\mathrm{jet}} e^{-\eta^{\mathrm{jet}}}}{2 y E_{e}}
$$

where now the sum runs over the two jets of highest $E_{T}^{\text {jet }}$. In the $x_{\gamma}^{\text {obs }}$ distribution thus obtained, the LO direct and resolved processes populate different regions, with the direct processes concentrated at high values of $x_{\gamma}^{\text {obs }}$. The peak arising from the direct contribution will not necessarily lie exactly at $x_{\gamma}^{\text {obs }}=1$ due to higher order effects and/or hadronization, but will still correspond to the kinematic region where most or all of the energy of the photon participated in the hardest scatter. Making a cut on $x_{\gamma}^{\text {obs }}$ provides a workable definition of direct and resolved events. Recall that beyond lowest order, such a separation is ambiguous. We introduce a similar definition for the proton, i.e.

$$
x_{p}^{\mathrm{obs}}=\frac{\sum_{\mathrm{jets}} E_{T}^{\mathrm{jet}} e^{-\eta^{\mathrm{jet}}}}{2 E_{p}} .
$$

In order to make our study as realistic as possible, we have performed jet finding on the hadronic final state using a cone algorithm. Unless stated explicitly otherwise, the cone radius used is $R=1$ (as has been used by both HERA experiments so far), and jets have $E_{T} \geq 6 \mathrm{GeV}$ 
and pseudorapidity $\eta=-\ln (\tan \theta / 2) \leq 2$. These cuts place our jets well within the region observable by the HERA detectors.

Multiple parton scattering is expected to affect jet rates in two ways. Firstly, and most obviously, the average number of jets per event should be increased when partons from secondary hard scatters are of sufficiently high $p_{T}$ to give jets in their own right. In addition, there is also the possibility that extra hard scatters can influence the observation of jets even when no parton from the secondary scatters is of high enough $p_{T}$ to produce an observable jet. Lower $p_{T}$ secondary scatters produce extra transverse energy in the event which can contribute to the pedestal energy underneath other jets in the event. In this way, the inclusion of multiple scattering can be thought of as an attempt to extend perturbative QCD in order to calculate some part of the so called 'soft underlying event', which is included in many simulations as a parameterized extrapolation of existing data. The first of these effects will only increase multi-jet cross sections, whilst leaving the inclusive jet cross section unchanged. The second effect will increase both inclusive and multi-jet cross sections. In figure 3, where inclusive jet (figure 3a,b) and dijet (figure 3 $\mathrm{c}, \mathrm{d}$ ) cross sections are shown (as a function of jet rapidity and mean jet rapidity respectively), it can be seen that the effect of multiple interactions is indeed more significant for the dijet cross sections than the inclusive jet cross sections. In all cases the effect is more significant at lower $E_{T}^{\mathrm{jet}}$. The direct photon contribution generated by HERWIG is included.

In table 11 the calculated rates for one-, two-, three- and four-jet events in the 'default model' are presented. The cross section for $m$ jets is defined here as the production cross section of events containing $m$ and only $m$ jets. There is an enhancement in all jet rates above the single jet rate and the significance rises with increasing jet multiplicity. Single jet events arise primarily from configurations where the second jet is forward of the $\eta$ cut. Without considering the extra transverse energy generated by multiple scattering, we would expect the sum of the jet cross sections obtained including multiple scattering to be lower than the corresponding sum obtained without multiple scattering (and the latter should be concentrated more at lower jet rates), by a factor equal to the mean multiplicity of jet pairs. This effect is mostly washed out by the large effect of the 'underlying event' generated by the secondary scatters. In this table only the contribution from LO resolved photon processes is included. Since the parton shower model without multiple scattering only generates multi-jet cross sections as a leading logarithmic dressing of the dijet cross section, it is only accurate in the region in which two jets are significantly harder than all the others. Therefore the predicted rates should only be taken as a rough indication. Nevertheless, since the logarithmically enhanced terms are included, the neglected terms are genuinely suppressed by powers of $\alpha_{s}$, without additional enhancement, whereas the eikonal expansion is an expansion (summed to all orders) in the large parameter $\alpha_{s} N^{2}$ (where $N^{2}$ is the product of the number densities). We therefore expect the enhancement of higher jet rates to remain significant.

These results imply that, even with these rather strict cuts on the jets, around 500 fourjet events (where the jets are easily observable in the detector) generated from multiple hard interactions should be present in the data already taken by each of the HERA detectors (around $10 \mathrm{pb}^{-1}$ by the end of 1995).

Figure 4 shows the $x_{\gamma}^{\text {obs }}$ and $x_{p}^{\text {obs }}$ distributions, again including the direct contribution generated using HERWIG (which can be seen peaking at high values of $x_{\gamma}^{\text {obs }}$ ). The inclusion of multiple scattering raises the cross section in the low $x_{\gamma}^{\text {obs }}$ region without affecting the high $x_{\gamma}^{\text {obs }}$ region, 


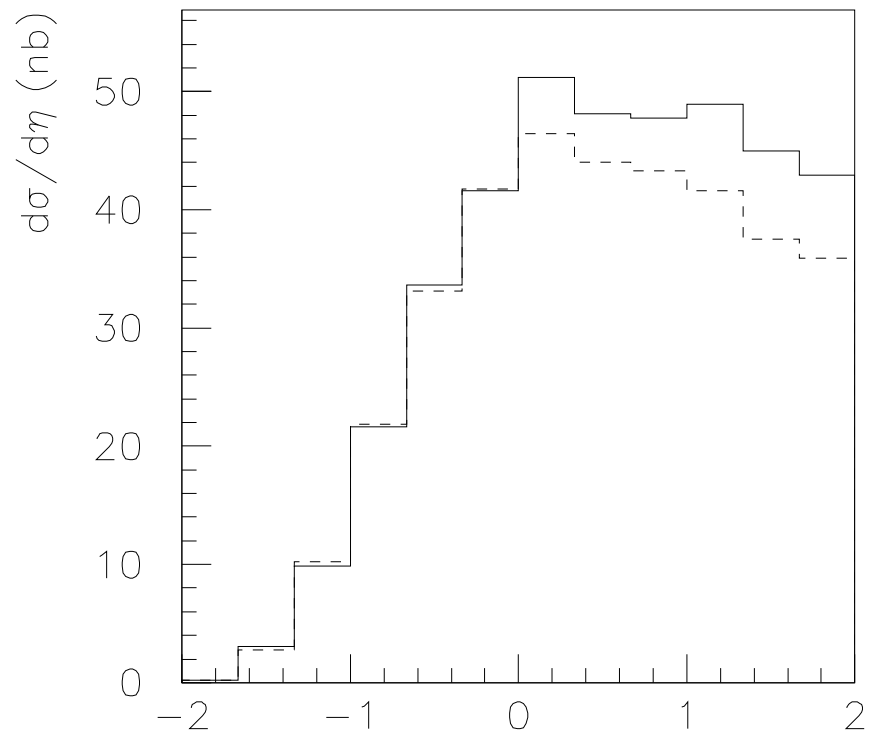

a)

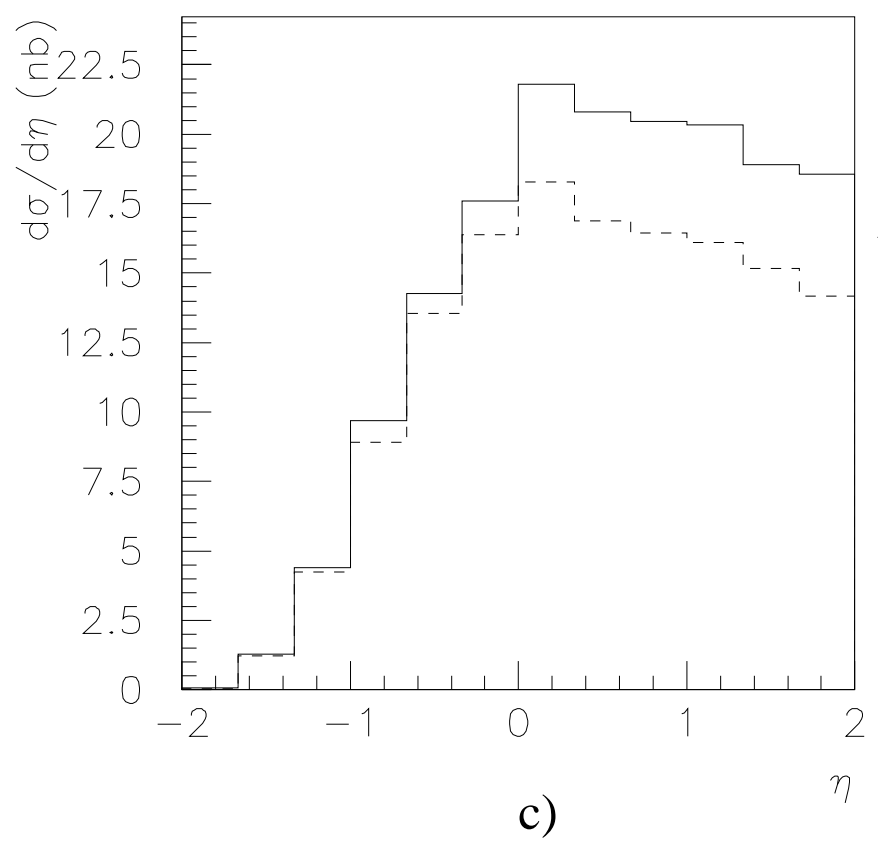

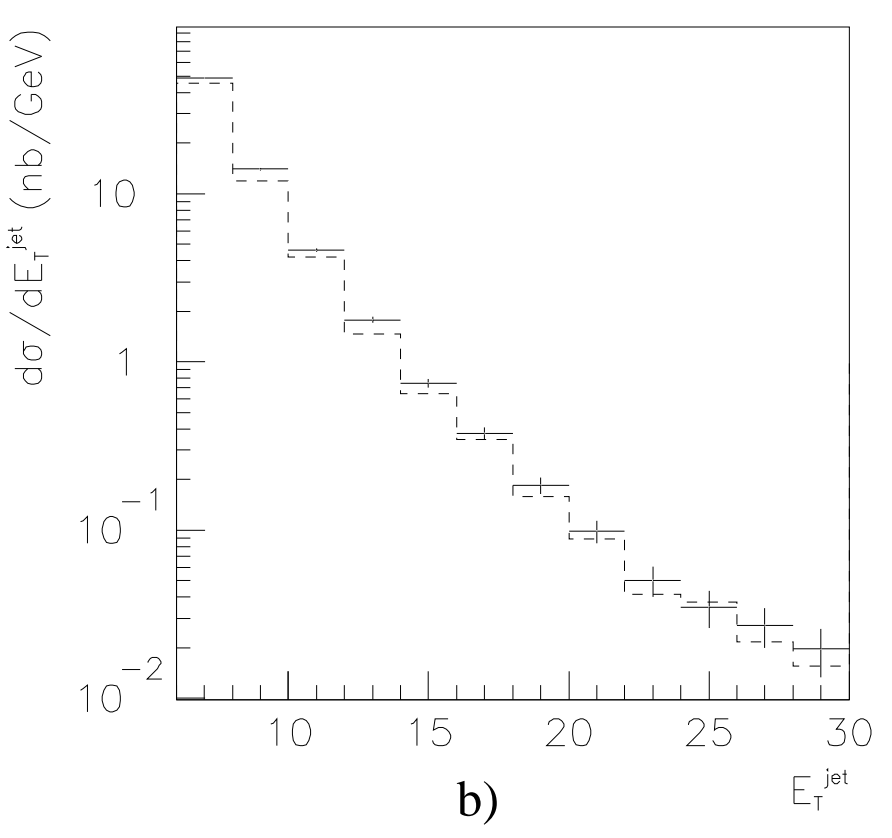

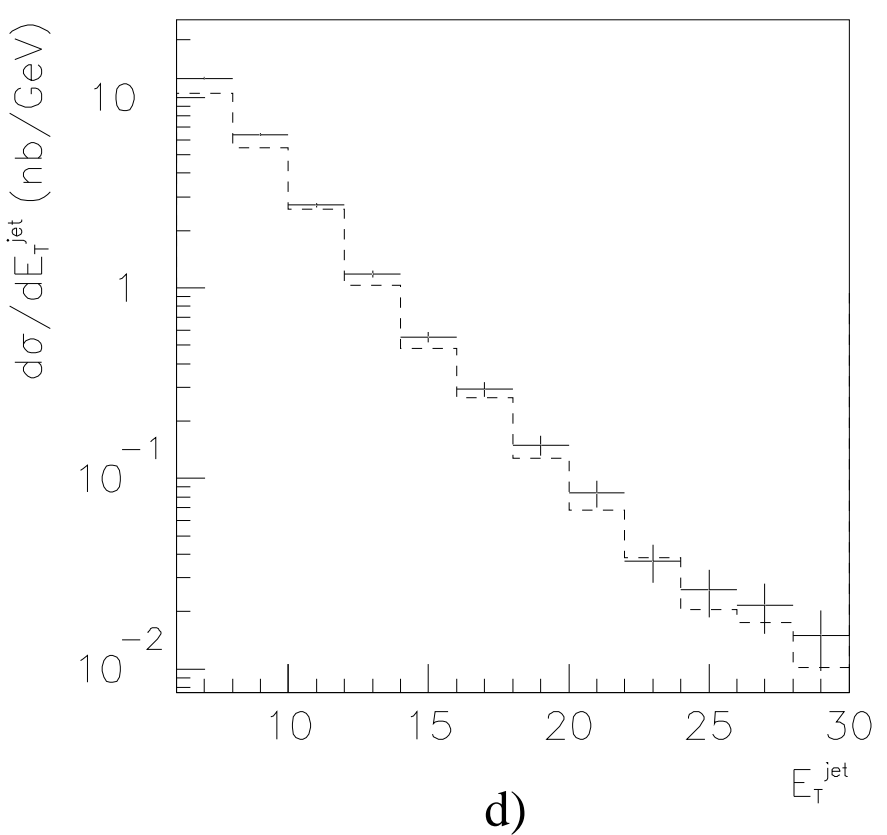

d)

Figure 3: Inclusive jet cross sections: a) $d \sigma / d \eta$ and b) $d \sigma / d E_{T}$. Dijet cross sections: c) $d \sigma / d \eta$ and d) $d \sigma / d E_{T}$. In all cases, $E_{T}^{\text {jet }}>6 \mathrm{GeV}$. The solid lines show the distributions when multiple scattering is included, the dashed lines show the distributions when no multiple scattering is allowed. In the $E_{T}^{\mathrm{jet}}$ plots, the statistical errors are indicated on the distribution for which multiple interactions are included. The errors on the 'no multiple interactions' distribution are similar. 


\begin{tabular}{|l|c|c|}
\hline & \multicolumn{2}{|c|}{ Cross section (nb) } \\
\hline & No multiple scatters & With multiple scatters \\
\hline One jet & $90.8 \pm 0.6$ & $89.9 \pm 0.8$ \\
\hline Two jet & $17.0 \pm 0.3$ & $19.5 \pm 0.4$ \\
\hline Three jet & $0.73 \pm 0.06$ & $1.15 \pm 0.09$ \\
\hline Four jet & $0.02 \pm 0.01$ & $0.05 \pm 0.02$ \\
\hline
\end{tabular}

Table 1: Resolved photon cross sections for multijet events. Jets have $E_{T}^{\text {jet }}>6 \mathrm{GeV}, \eta<2$, $R=1$ and the proton and photon parton distribution sets used are GRV and GS2 respectively. The errors shown are statistical.
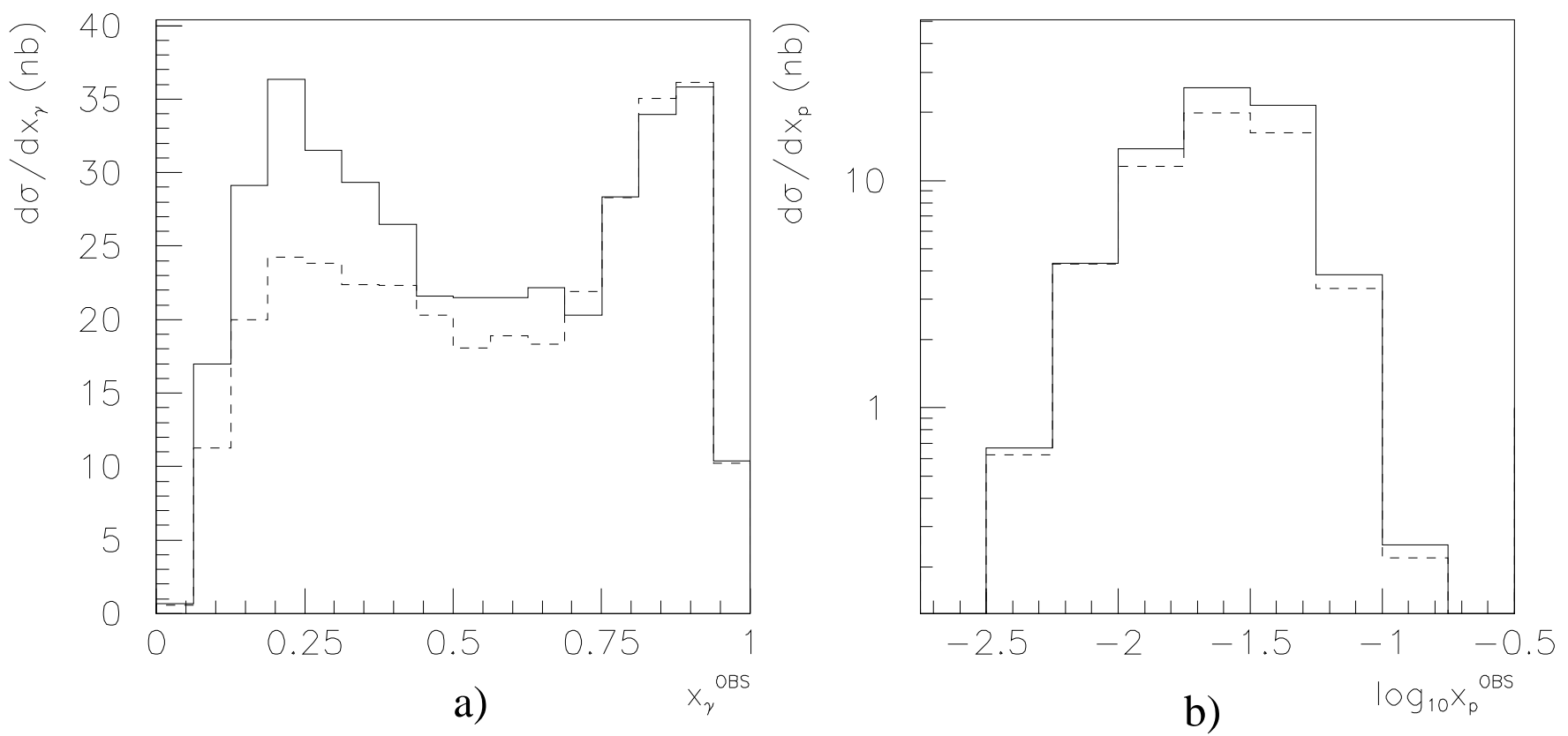

Figure 4: a) $d \sigma / d x_{\gamma}^{\text {obs }}$ b) $d \sigma / d x_{p}^{\text {obs }}$ The solid histograms show the distributions when multiple scattering is included, the broken histograms show the distributions when multiple scattering is neglected. 


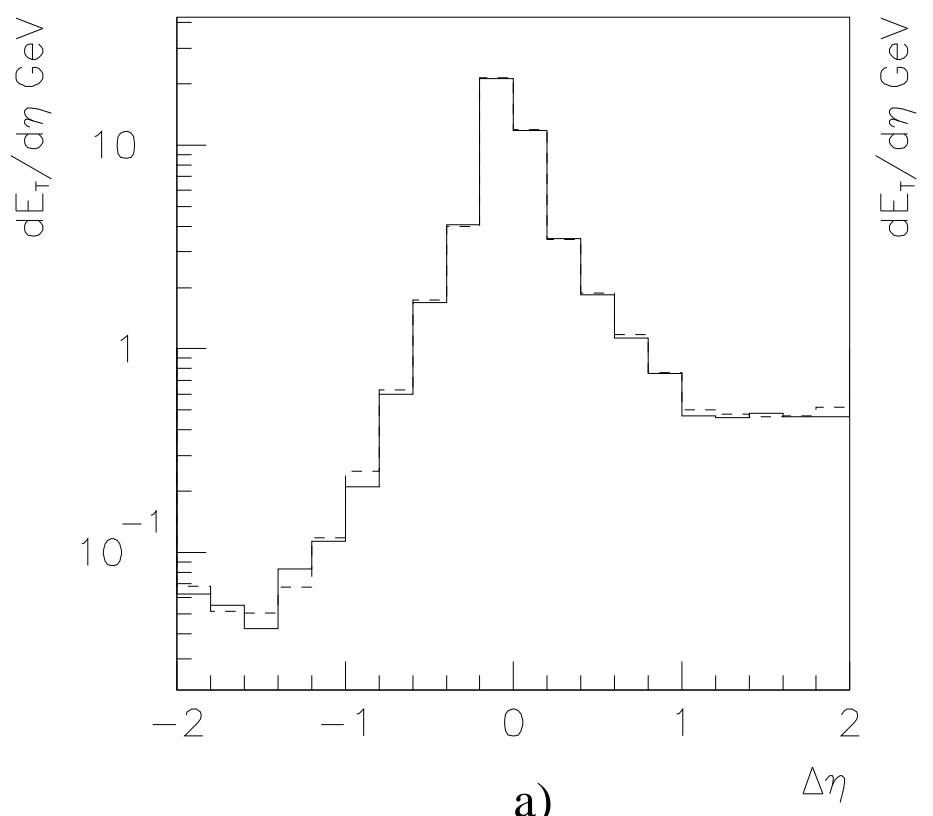

a)

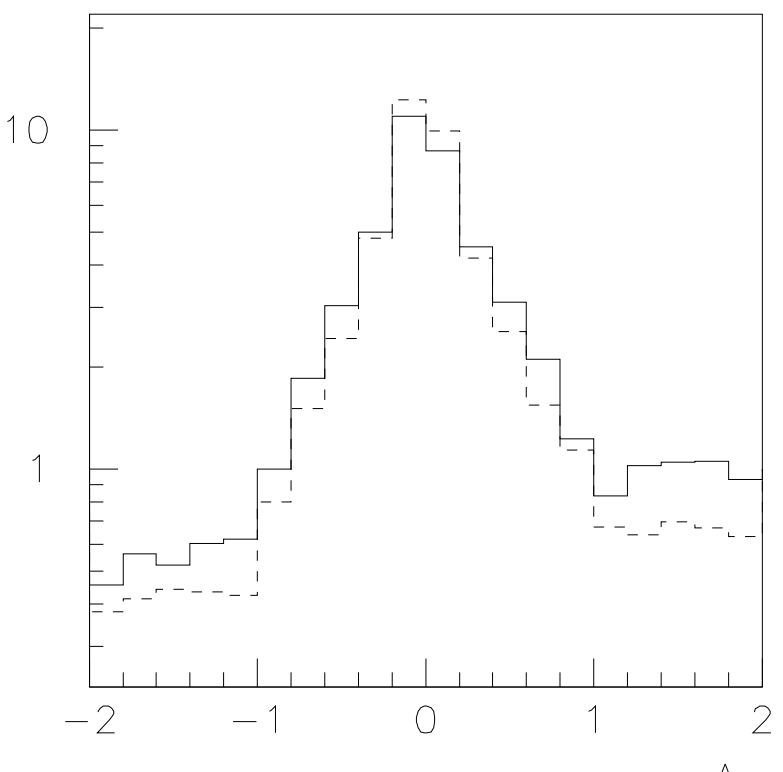

b)

Figure 5: Transverse energy flow as a function of $\Delta \eta$ integrated over $|\Delta \phi|<1.0$ for jets with $E_{T}>6 \mathrm{GeV}$ in the range $-2.0<\eta<2.0$ and $x_{\gamma}^{\text {obs }} \geq 0.75$ (a) and $x_{\gamma}^{\text {obs }}<0.75$ (b). The solid lines show the distributions when multiple scattering is included, the dashed lines show the distributions when no multiple scattering is allowed.

which is dominated by direct photoproduction. In the (uncorrected) $x_{\gamma}^{\text {obs }}$ distributions presented in refs. [2, 3] there is an excess of data over the standard simulations in just this region. Multiple interactions may have a role to play in resolving this discrepancy. The effect of multiple interactions can be seen to be largest for the $x_{p}$ values in the middle of the available range. This is easy to understand since the lower $x_{p}$ values are strongly correlated to large $x_{\gamma}$ values (where direct photon interactions are dominant).

Figure 5 shows how multiple scattering affects the profiles of jets in the $\eta$ - $\phi$ plane. In particular we look at jets in both the high and low $x_{\gamma}^{\text {obs }}$ regions.

The effect of this model for multiple interactions on the jet profiles is significant but fairly small. In the high $x_{\gamma}^{\text {obs }}$ region the effect is negligible. In the low $x_{\gamma}^{\text {obs }}$ case, there is a slight broadening of the jet and an increase in the pedestal of energy flow around the jet by around $300 \mathrm{MeV}$ per unit of $\eta$ on the forward (proton) side of the jet and by around $100 \mathrm{MeV}$ on the rear (photon) side. In measured energy flows at HERA, an excess of energy flow in the forward region has been observed [25] and shown to occur principally at low values of $x_{\gamma}^{\text {obs }}$ [3]. None of the jet profiles presented by the HERA experiments are corrected for detector effects, and so smearing of jet and particle energies makes direct comparison impossible at present. One might take the view that the jet profile data could be used to tune $p_{T}^{\min }$. This is not a very sensible thing to do, since (as we shall see) uncertainties in the photon gluon density can easily be traded off against the value of $p_{T}^{\min }$. 


\begin{tabular}{|l|c|c|}
\hline & \multicolumn{2}{|c|}{ Cross section (nb) } \\
\hline & No multiple scatters & With multiple scatters \\
\hline One jet & $93.2 \pm 1.0$ & $124.4 \pm 1.2$ \\
\hline Two jet & $15.0 \pm 0.4$ & $28.3 \pm 0.6$ \\
\hline Three jet & $0.62 \pm 0.08$ & $2.13 \pm 0.16$ \\
\hline Four jet & $0.01 \pm 0.01$ & $0.15 \pm 0.04$ \\
\hline
\end{tabular}

Table 2: As Table 1, but using LAC1 parton distribution functions for the photon instead of GS2.

\section{Sensitivity to Parton Distributions}

The fact that jet cross sections, and in particular dijet cross sections, are affected by the presence of multiple hard interactions means that these cross section may be misleading if used to distinguish between different parton distributions in the proton and/or photon without giving due consideration to the effects of multiple interactions. In figure we show the $x_{\gamma}^{\text {obs }}$ distribution and the low- $x_{\gamma}^{\text {obs }}$ jet profiles obtained using the GS2, LAC1 and GRV parton distribution sets for the photon.

In general, for this kinematic region, the effect of multiple scattering is greater than or similar to the changes in the cross sections produced by using different parton distributions. This is particularly notable in the jet profiles, which in the absence of multiple interactions are insensitive to the photon parton distribution, but which depend upon it significantly when multiple interactions are allowed. The effect of multiple interactions is most pronounced if the LAC1 parton distribution set is used. This is due to the high density of low- $x_{\gamma}$ gluons present in the parameterization. In table 2 we show the rates of multijet events for the LAC1 photon parton distribution set, where it can be seen that the multijet rates are massively enhanced by multiple interactions.

\section{$5 \quad$ Variations on the Default Model}

Despite the fact that it is a reasonably simple and well defined procedure, there are of course several uncertainties and free parameters in the model. These include the values of $p_{T}^{\min }, P_{\text {res }}$, and $\nu^{2}$. The parameter which has the strongest effect of the cross sections is the value of $p_{T}^{\min }$. Lowering $p_{T}^{\min }$ to $2 \mathrm{GeV}$ dramatically increases the effect of multiple interactions, as shown by the effect on the jet profiles and and $x_{\gamma}^{\text {obs }}$ distribution in figures 7 a and c. The energy flow around the jet axis and the cross section at low $x_{\gamma}^{\text {obs }}$ increase when $p_{T}^{\min }$ is lowered. This occurs whether or not multiple interactions are included, but in the presence of multiple interactions the effect is much greater.

The value of $P_{\text {res }}$ used in our 'default model' is around the lowest sensible value. Increasing it from $1 / 300$ to $1 / 150$ decreases the effect of multiple scattering since we now correspondingly reduce the number density of resolved partons. Since $P_{\text {res }}$ is the probability that a photon is resolved, its value is closely related to the photon's parton distribution functions. Indeed, if one fixed the parton densities in a resolved photon and only varied $P_{\text {res }}$, there would be no variation in the effect of multiple interactions. However, the biggest differences between current sets is 


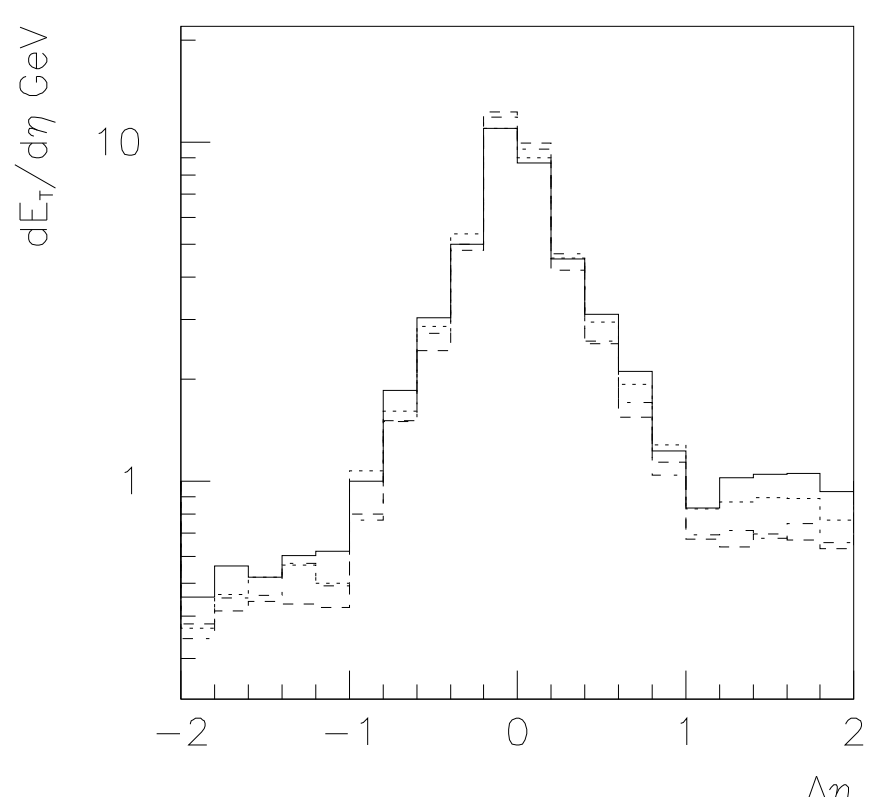

a)

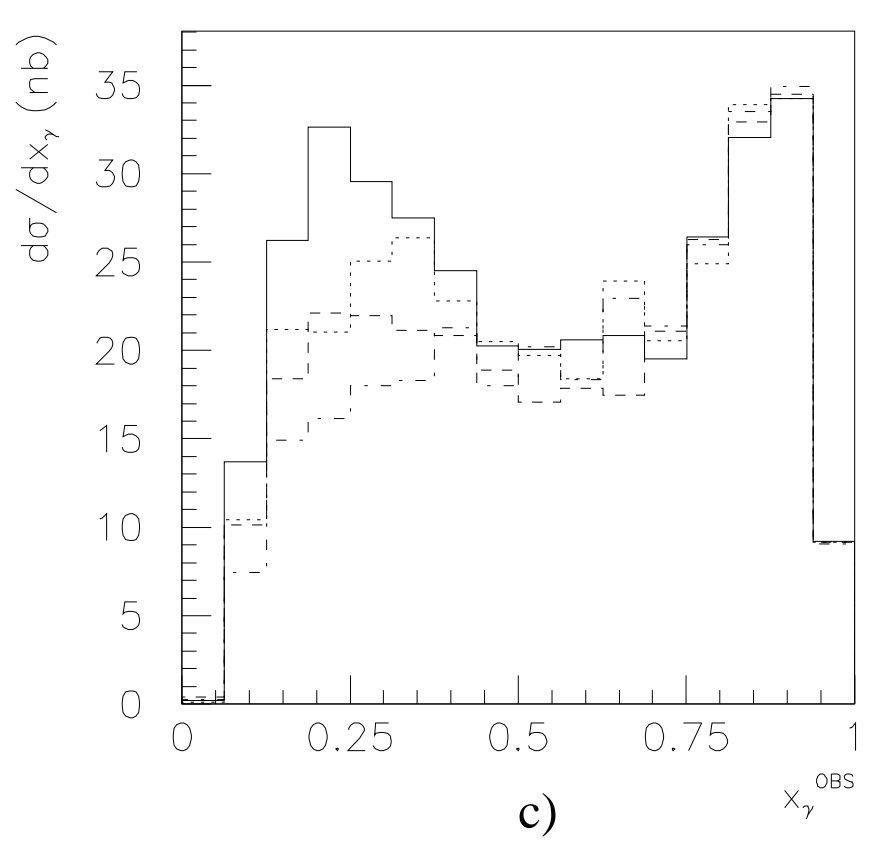

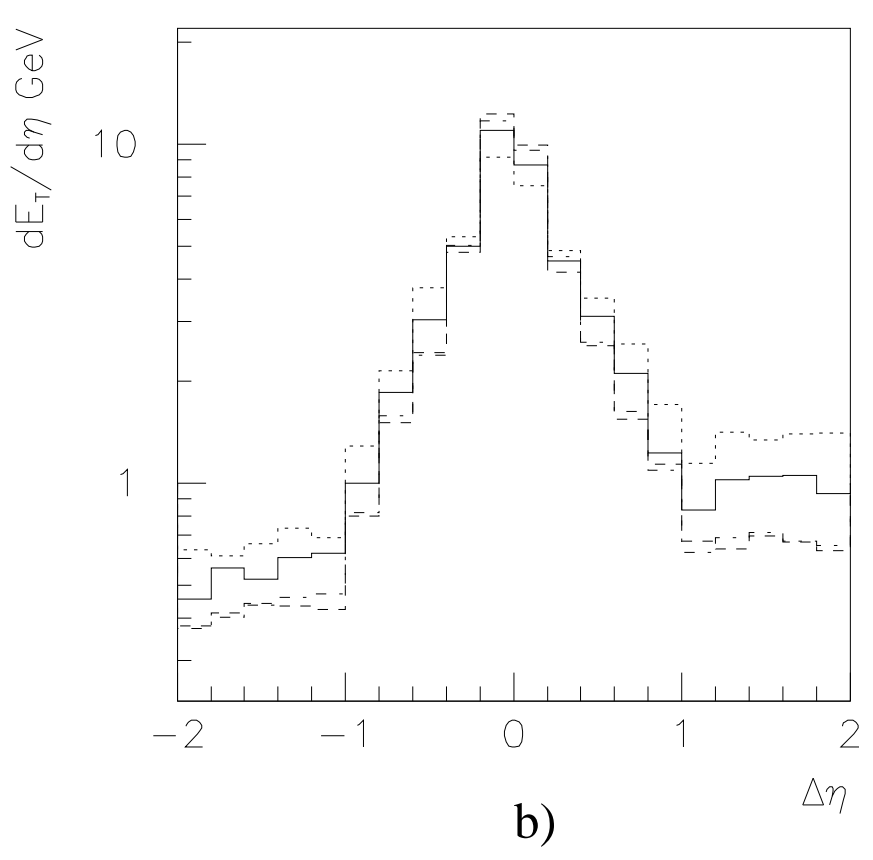

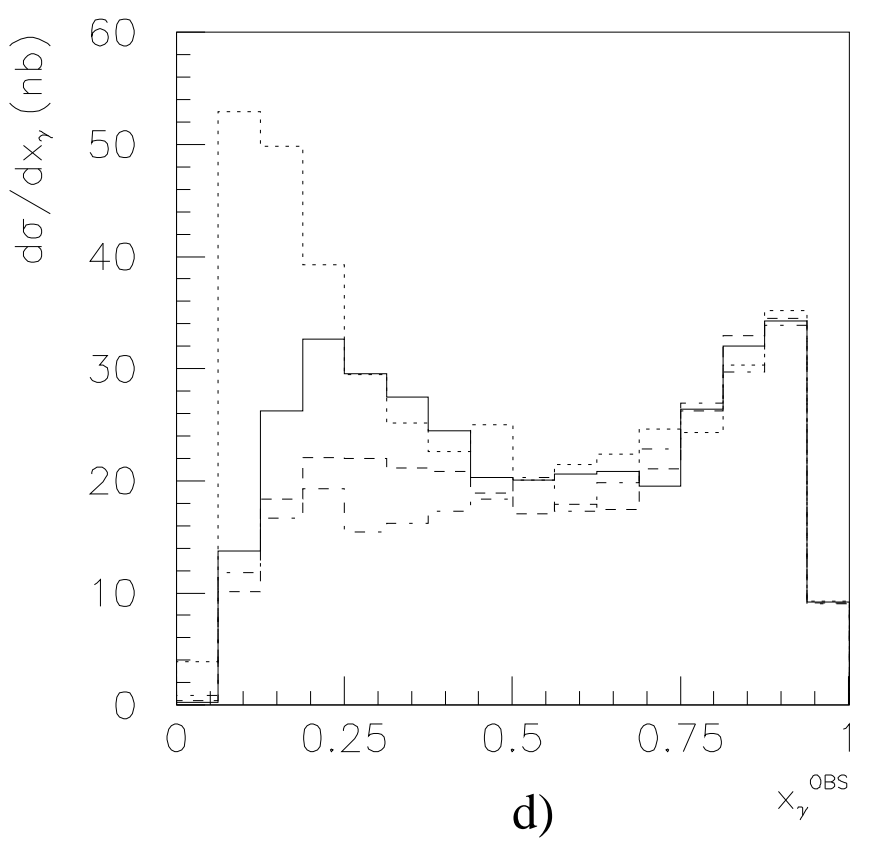

Figure 6: Effect of different photon parton distribution sets: a) and b) show transverse energy flow as a function of $\Delta \eta$ integrated over $|\Delta \phi|<1.0$ for jets with $E_{T}>6 \mathrm{GeV}$ in the range $-2.0<\eta<2.0$ and $x_{\gamma}^{\text {obs }} \leq 0.75$. The solid and dashed lines show the distributions obtained using the GS2 photon parton densities with and without multiple interaction respectively. In a) the dotted and dash-dotted lines show the distributions obtained using the GRV photon parton densities with and without multiple interactions respectively, and in b) they show the same distributions obtained using the LAC1 photon parton densities. Figures c) and d) show the $x_{\gamma}^{\text {obs }}$ cross section. The lines in c) have the same meaning as in a), and those in d) have the same meaning as in b). In all cases the proton parton distribution set is taken from GRV. 
in the distribution functions within a resolved photon, rather than in the value of $P_{\text {res }}$ used. Therefore most of the differences shown in section 4 would remain even if $P_{\text {res }}$ was adjusted to the values in the distribution functions.

Changing the value of $\nu^{2}$ from $0.47 \mathrm{GeV}^{2}$ to $1.5 \mathrm{GeV}^{2}$ makes the photon more compact, i.e. it increases the resolved $-\gamma$ number densities at small impact parameters whilst decreasing them at large impact parameters. Since peripheral $\gamma p$ collisions are rarer, the net result is to increases the effect of multiple interactions for each resolved $-\gamma-p$ interaction but to make the cross section for such reactions smaller. The effects of these changes on the jet profiles and $x_{\gamma}^{\text {obs }}$ distribution are shown in figures $7 \mathrm{~b}$ and $\mathrm{d}$.

\section{Comparison with Data}

Both ZEUS [25] and H1 [26] have published inclusive jet cross sections for photoproduction at HERA, and ZEUS has also published dijet cross sections [3]. Our default multiple interactions model is compared to a selection of this available data in figure 8 .

The dijet cross sections are measured as a function of the average $\eta$ of the two jets $(\bar{\eta})$ for direct and resolved photoproduction (defined by a cut at $x_{\gamma}^{\text {obs }}=0.75$ ) for $E_{T}^{\text {jet }}>6 \mathrm{GeV}$, $P^{2}<4 \mathrm{GeV}^{2}, 0.2<y<0.8$ and $|\Delta \eta|<0.5$ ( $P^{2}$ is the photon virtuality). There is a correlated uncertainty due to the calorimeter energy scale of around $20 \%$ in the direct and $25 \%$ in the resolved data, which here we have added in quadrature to the other systematic errors. The agreement with the direct dijet cross section is good in all cases, confirming that the separation based upon $x_{\gamma}^{\text {obs }}$ removes to a large extent any sensitivity of this cross section to the photon structure (and hence multiple interactions). In the case of the resolved dijet cross section, the calculations are in general too low, as was also the case for the analytic LO QCD calculations presented in [3]. Multiple interactions raise the calculated cross section towards the data and, given the large systematic errors on the data (in particular the overall normalization uncertainty) perhaps the disagreement is not so significant yet. A major source of the systematic errors in the data is the discrepancy in the jet profiles between data and Monte Carlo models, it is to be hoped that improvements in the simulations (of which the inclusion of multiple interactions is an example) will contribute to the reduction of these errors in future measurements.

The inclusive single jet measurements of $\mathrm{H} 1$ and ZEUS are made over different $y$ ranges $(0.2<$ $y<0.85$ for ZEUS and $0.25<y<0.7$ for H1), different $P^{2}$ ranges $\left(P^{2}<4 \mathrm{GeV}^{2}\right.$ for ZEUS and $P^{2}<0.01$ for H1) and different $E_{T}^{\text {jet }}$ ranges $\left(E_{T}^{\text {jet }}>8 \mathrm{GeV}\right.$ for ZEUS and $E_{T}^{\text {jet }}>7 \mathrm{GeV}$ for $\mathrm{H} 1)$. There is a correlated uncertainty due to the calorimeter energy scale of around $20 \%$ in the ZEUS inclusive jet data, and a $25 \%$ overall normalization uncertainty in the H1 data. In both cases we have here added these in quadrature to the other systematic errors. In addition it is worth noting here that since our model uses only the LO matrix elements plus parton showers, there is an expected uncertainty in the calculations due principally to the choice of scale. In the calculations available for these processes, the NLO corrections have been as high as $40 \%$ [27 although, as mentioned earlier, the parton shower would be expected to reproduce at least part of this correction. Since the ZEUS data are collected up to larger values of $y$, multiple interactions are expected to have a larger effect and this is seen by comparing the solid and dotted curves in figure 8c with the corresponding curves in figure 8d. The broken lines, 


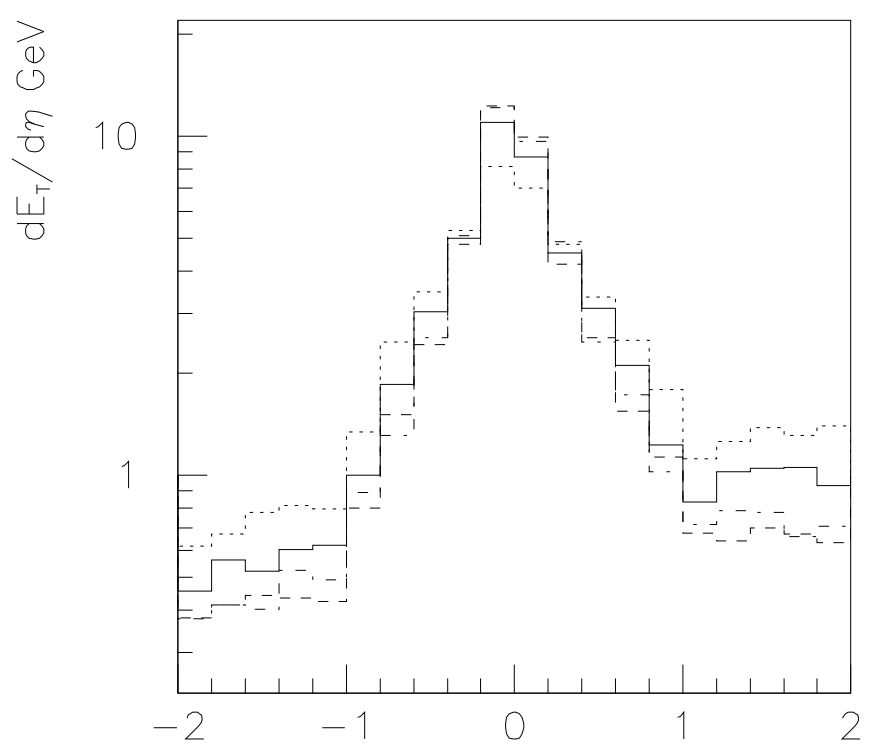

a)

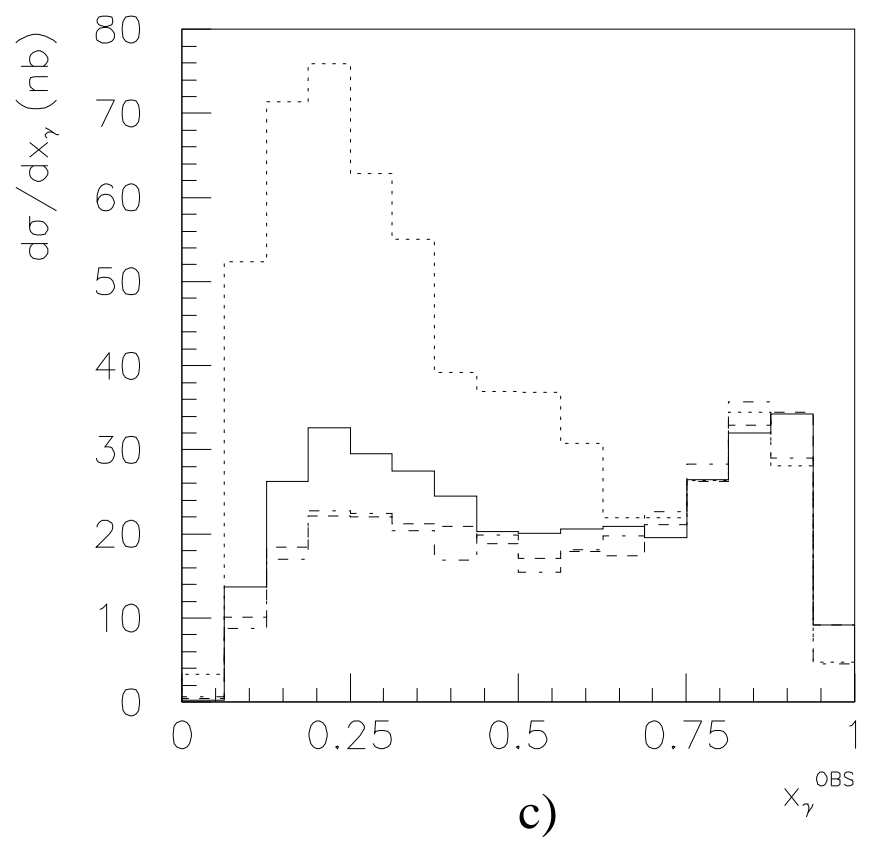

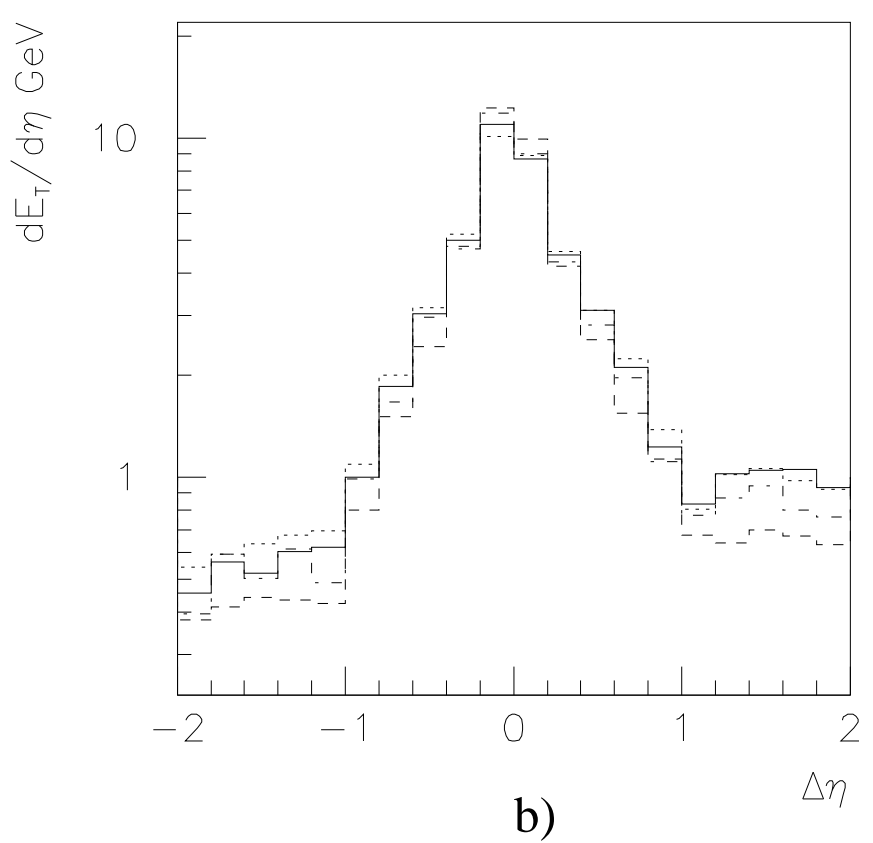

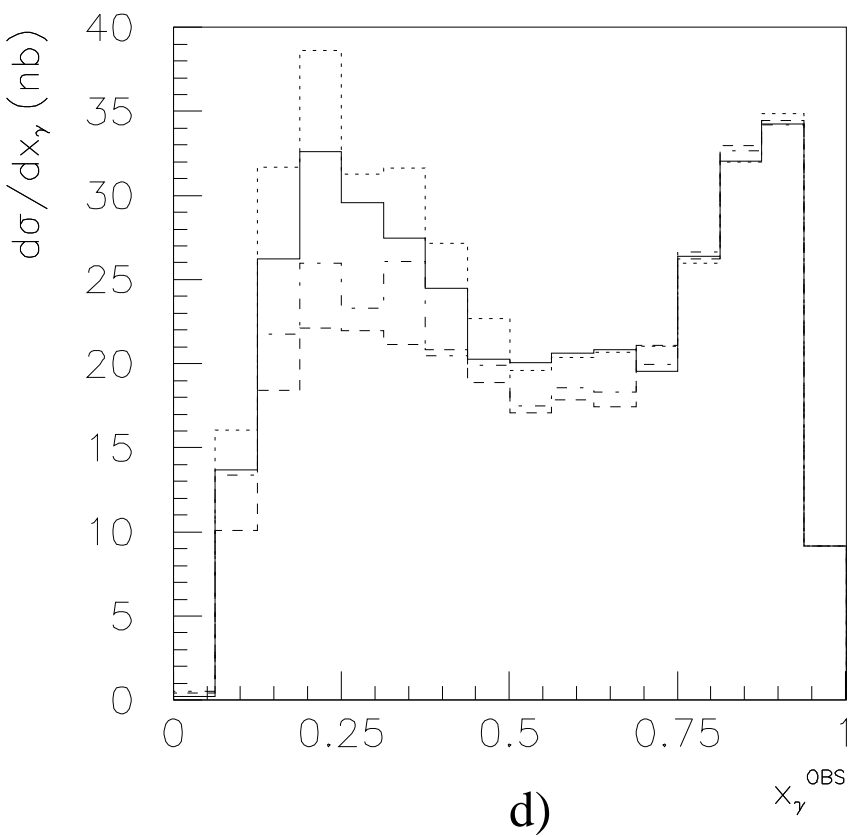

Figure 7: Sensitivity to model parameters: a) and b) show transverse energy flow as a function of $\Delta \eta$ integrated over $|\Delta \phi|<1.0$ for jets with $E_{T}>6 \mathrm{GeV}$ in the range $-2.0<\eta<2.0$ and $x_{\gamma}^{\text {obs }} \leq 0.75$. In all cases, the solid and dashed lines show the distributions obtained with and without multiple interactions respectively in the default model). In a) the dotted and dashdotted lines show the distributions obtained with and without multiple interactions respectively, when $p_{T}^{\min }$ is lowered from $3 \mathrm{GeV}$ to $2 \mathrm{GeV}$. In b) the dotted line shows the distribution obtained with multiple interactions when the photon radius $\nu^{2}$ is changed to $1.5 \mathrm{GeV}^{2}$ and the dashdotted lines show that obtained with $P_{\text {res }}=1 / 150$. Figures c) and d) show the $x_{\gamma}^{\text {obs }}$ cross section. The lines in c) have the same meaning as in a), and those in d) have the same meaning as in b). 


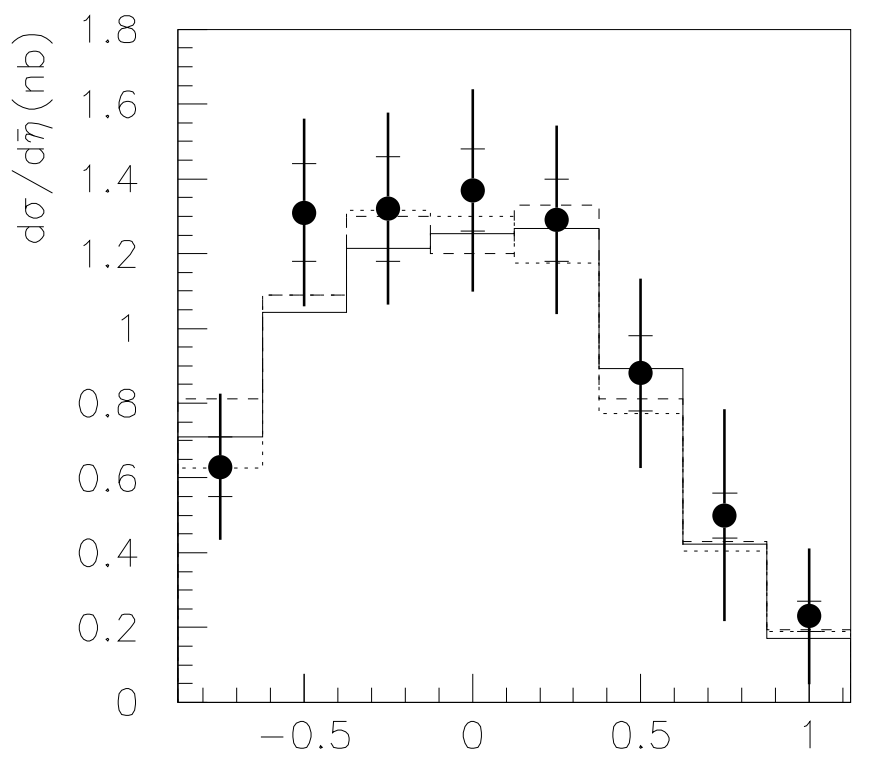

a)

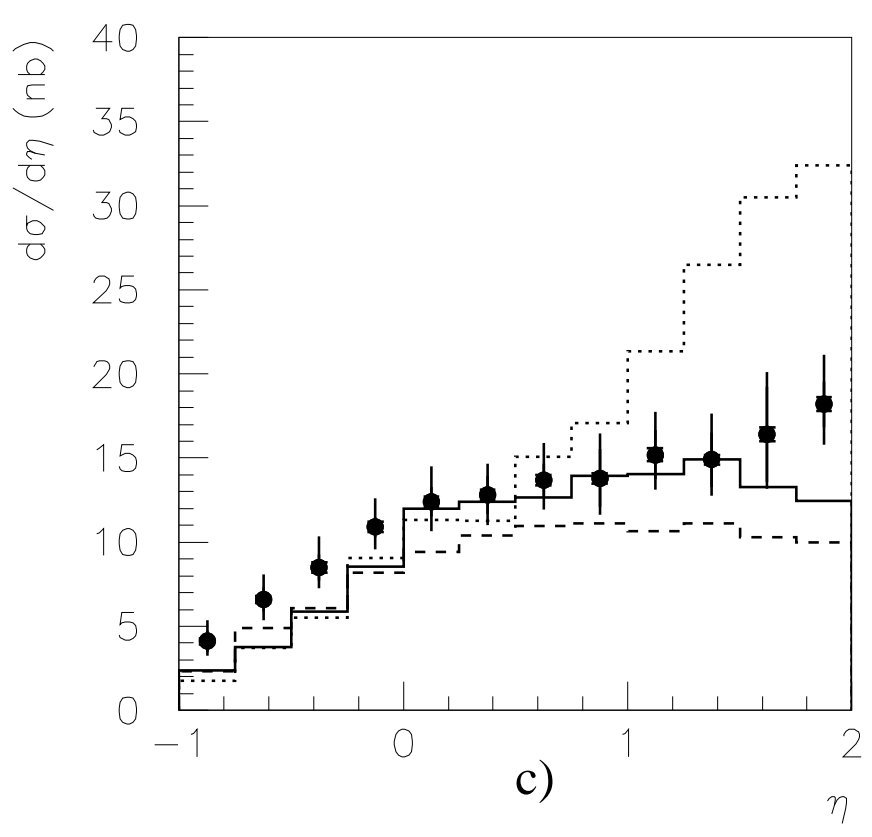

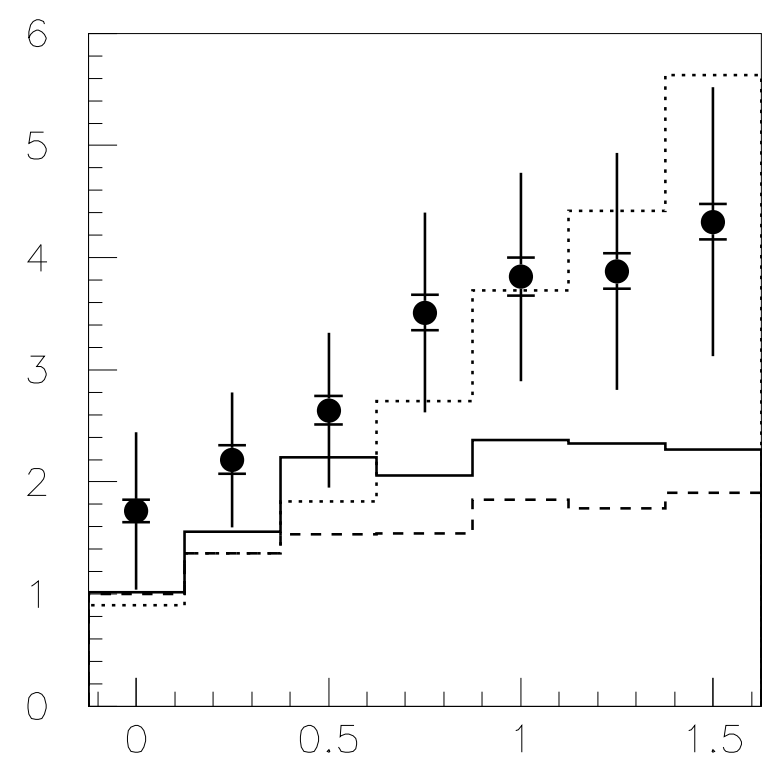

b)

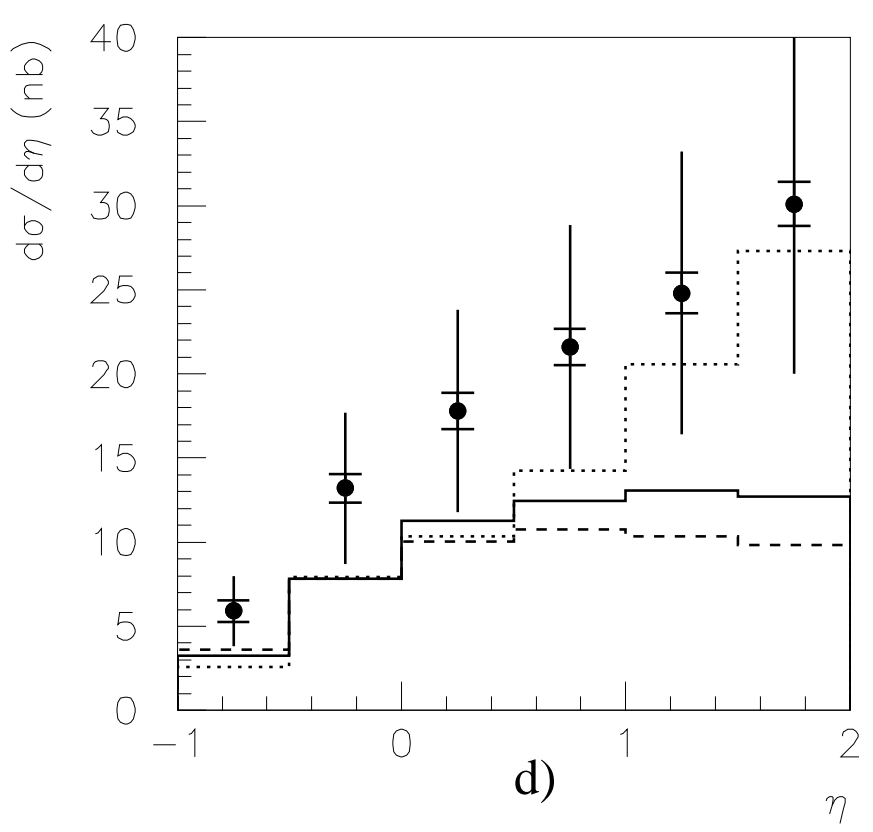

Figure 8: ZEUS data compared with HERWIG. a) and b) show the differential dijet cross sections for direct and resolved photon events respectively (where the separation is defined by a cut on $x_{\gamma}^{\text {obs }}$ ) by the ZEUS collaboration [3]. c) and d) shows the differential inclusive jet cross section as a function of $\eta^{\text {jet }}$ as measured by the ZEUS [25] and H1 [26] collaborations respectively. The inner error bars indicate the statistical uncertainty, and the outer one the statistical and systematic errors added in quadrature. In all cases the solid histogram shows the calculation of the default multiple interaction model, the dotted line shows the calculation using the default but using LAC1 instead of GS2 for the parton distribution set, and the dashed line shows the result of HERWIG with no multiple interactions. 
corresponding to the HERWIG predictions without multiple scattering are very similar (the effect of increasing the $P^{2}$ range in the ZEUS data is compensated by the lower $E_{T}^{\text {jet }}$ cut of $\mathrm{H} 1$ ). Given the uncertainties mentioned above, we should be cautious in drawing any conclusions from these data. However, there is a suggestion - at the $(1 \sim 2) \sigma$ level - that the HERA data may be incompatible, in that $\mathrm{H} 1$ inclusive jets agree better with LAC1 distribution (or very significant multiple interaction effects) whilst the ZEUS inclusive jet data lie closer to the result of our default multiple interaction model.

Bearing in mind the above discussions, there are signs that including multiple interactions can bring the model into better agreement with existing data in those regions where discrepancies exist (i.e. the forward region in both dijet and inclusive jet production), whilst having a small effect on those regions (i.e. direct dijet, low $\eta^{\text {jet }}$ inclusive) where the agreement is already good.

\section{Conclusions}

We have performed a detailed study of the unitarization corrections which are expected to appear in high energy $\gamma p$ interactions. We used an eikonal approach to model their effects which, in the perturbative domain, manifest themselves through the appearance of multiple parton scattering. By performing a Monte Carlo simulation of our model we have been able to make a study of the detailed properties of the hadronic final state which enables us to make use of the vast amount of HERA data on photoproduction. We find that, for partons produced with $p_{T}>3 \mathrm{GeV}$, over $4 \%$ of events can be expected to contain more than one hard process at typical HERA energies.

For reasonable experimental cuts the effect of multiple parton interactions on measured jet cross sections could be significant. The size of the effect is expected to depend strongly upon the parton distributions in the photon and can be as high as 100\% in some regions for dijet cross sections already measured at HERA. The effect is even more significant for higher jet rates, leading to an overall enhancement of a factor of up to around five in the four jet rate (with all four jets contained in a detector). These events should provide a means of unambiguously discovering or ruling out some multiple interaction models in the near future, although untangling a clear signature in a realistic multi-hadron final state remains something of a challenge.

The model considered here also leads to the generation of a 'semi-hard' underlying event. This affects the energy flow forward of the jet direction, as do other models which are already being used to to describe HERA data [18, 28], where the energy flow in the forward (proton) direction is poorly reproduced in simulations which do not include multiple interactions.

Collectively, the effects of multiple interactions may well make the extraction of photon parton distribution functions (especially the gluon) rather difficult.

The Monte Carlo program used here runs in conjunction with HERWIG version 5.8 and is available from the authors. Further information can be obtained from the world wide web page http://surya11.cern.ch/users/seymour/herwig/

\section{Acknowledgements}

It is a pleasure to thank DESY (where much of this work was performed) for their hospitality. For useful discussions we thank Greg Feild, Lutz Feld, John Storrow and Rod Walker. 


\section{References}

[1] H1 Collaboration, T. Ahmed et al, Phys. Lett. B297 (1992) 205.

ZEUS Collaboration, M. Derrick et al, Phys. Lett. B297 (1992) 404.

[2] ZEUS Collaboration, M. Derrick et al, Phys. Lett. B322 (1994) 287.

[3] ZEUS Collaboration, M. Derrick et al, Phys. Lett B348 (1995) 665.

[4] J. R. Forshaw and R. G. Roberts, Phys. Lett. B319 (1993) 539.

[5] M. Klasen and G. Kramer, DESY 95-159; DESY 95-226.

[6] ZEUS Collaboration, M. Derrick et al, Phys. Lett. B316 (1993) 412.

H1 Collaboration, I. Abt et al, Nucl. Phys. B407 (1993) 515.

ZEUS Collaboration, M. Derrick et al, Z. Phys. C65 (1995) 379.

[7] J. C. Collins and G. A. Ladinsky, Phys. Rev. D43 (1991) 2847.

[8] J. R. Forshaw and J. K. Storrow, Phys. Lett. B268 (1991) 116; erratum B276.

[9] R. S. Fletcher et al, Phys. Rev. D45 (1992) 337.

[10] G. Marchesini et al, Comp. Phys. Comm. 67 (1992) 465.

[11] H1 Collaboration, T.Ahmed et al, Z. Phys. C69 (1995) 27.

ZEUS Collaboration, M.Derrick et al, Z. Phys. C63 (1994) 391.

[12] A. Donnachie and P. V. Landshoff, Phys. Lett. B296 (1992) 227.

[13] J.R.Forshaw and J.K.Storrow, Phys.Rev. D46 (1992) 4955; Phys.Lett. B321 (1994) 151.

K.Honjo et al, Phys.Rev. D48 (1993) 1048.

R.S.Fletcher, T.K.Gaisser and F.Halzen, Phys.Lett. B298 (1993) 442.

G.A.Schuler and T.Sjöstrand, Nucl.Phys. B407 (1993) 539.

M.Drees, hep-ph/9406414, in the proceedings of the Workshop on Two-Photon Physics at LEP and HERA, Lund, May 1994.

[14] M.Drees and R.Godbole, Pramana J.Phys. 41 (1993) 83.

[15] L.Durand et al, Phys.Rev. D47 (1993) R4815.

[16] J. M. Butterworth and J. R. Forshaw, J. Phys. G19 (1993) 1657.

J. M. Butterworth and J. R. Forshaw, RAL 94-088, in the proceedings of the Workshop on Two-Photon Physics at LEP and HERA, Lund, May 1994.

[17] J.M.Butterworth et al, CERN-TH/95-83, to appear in the proceedings of the 'Photon 95' conference, Sheffield, April 1995.

[18] T. Sjöstrand and M. van Zijl, Phys.Rev. D36, 2019 (1987).

[19] L. E. Gordon and J. K. Storrow, Z. Phys. C56 (1992) 307. 
[20] M. Glück, E. Reya and A. Vogt, Z. Phys. C53 (1992) 127;

M. Glück and E. Reya, Dortmund DO-TH 93/27 (1993);

M. Glück, E. Reya and A. Vogt, Phys. Lett B306 (1993) 391;

M. Glück, E. Reya and A. Vogt, Z. Phys. C67 (1995) 433.

[21] V.N.Gribov and L.N.Lipatov, Sov.J.Nucl.Phys. 15 (1972) 438.

L.N.Lipatov, Sov.J.Nucl.Phys. 20 (1974) 94.

Y.L.Dokshitzer, Sov.Phys. JETP 46 (1977) 641.

G.Altarelli and G.Parisi, Nucl.Phys. B126 (1977) 298.

[22] G. Marchesini and B.R. Webber, Nucl. Phys. B238 (1984) 1; Nucl. Phys. B310 (1988) 461.

[23] T. Sjöstrand, Phys. Lett. 157B (1985) 321.

[24] B.R. Webber, Nucl. Phys. B238 (1984) 492.

[25] ZEUS Collaboration, M. Derrick et al, Phys. Lett. B322 (1994) 287.

[26] H1 Collaboration, S. Aid et al, DESY 95-219.

[27] M.Klasen, G.Kramer and S.G.Salesch, Z.Phys C68 (1995) 113.

[28] R.Engel, Z.Phys. C66 (1995) 203. 